1 Hacettepe Journal of Mathematics and Statistics

$\bigcap$ Volume 46 (6) (2017), 1069-1075

\title{
On partial metric spaces and partial cone metric spaces
}

\author{
Seithuti Moshokoa*
}

\begin{abstract}
It this article we shall show that partial metric spaces and partial cone metric spaces are quasi-uniformizable and hence quasi-metrizable. Finally, an application to the Banach's fixed point theorem will be presented in this context.
\end{abstract}

Keywords: Partial metric space, Partial cone metric space, quasi metric space, Quasi uniform space, Fixed point.

2000 AMS Classification: Primary 54E35, Secondary 54A10, 54350, 54H25.

Received: 08.03.2016 Accepted : 24.03.2017 Doi : 10.15672/HJMS.2017.461

\section{Introduction}

In applications, for instance in the study of denotational semantics of programming languages some topological models are constructed. It so happen that such a model need not be a Hausdorff space but a $T_{0}$-space [9]. An ideal candidate for such a tool is partial metric spaces. In the article we study topological properties of partial metric spaces, partial cone metric spaces and quasi metric spaces.

The notion of a partial metric space was introduced by $\mathrm{S} G$ Matthews [9], since then many interesting results were presented in the literature, see references in [9] and also those at the end of this article. The focus ranges from topological properties of these spaces to applications to theoretical computer science including generalizations of Banach' fixed point principle from metric spaces to partial metric spaces. In this paper we further study topological properties of partial cone metric spaces via quasi-uniform spaces. In this way we establish a relationship between partial cone metric spaces and quasi-metric spaces. Furthermore we show that both partial cone metric spaces and partial metric spaces are quasi-uniformizable.

${ }^{*}$ Department of Mathematics and Statistics, Tshwane University of Technology, Pretoria, 0008, South Africa.

Email : MoshokoaSP@tut.ac.za 


\section{Preliminaries}

We refer the reader to ([9], [11]) for more details and the connection of partial metric spaces to theoretical computer science.

Let $X$ be a nonempty set. A function $d: X \times X \rightarrow[0, \infty)$ satisfying for all $x, y, z \in X$ :

(i) $d(x, x)=0$

(ii) $d(x, y) \leq d(x, z)+d(z, y)$ is called quasi-pseudo metric on $X$ and the pair $(X, d)$ is called a quasi-pseudo metric space. If the function $d$ satisfies $(i),($ ii $)$, and $($ iii $) d(x, y)=$ $d(y, x)$ then, $d$ is called a pseudo metric and the pair $(X, d)$ is referred to as a pseudo metric space. It is well-known that a pseudo metric on $X$ is a metric if $d(x, y)=0$ is equivalent to $x=y$. Now a quasi-pseudo metric that satisfies $(i)^{\prime} d(x, y)=0=d(y, x)$ if and only if $x=y$ is referred to as a quasi metric on $X$. Clearly every metric on $X$ is a pseudo metric but not conversely. When $d$ is a pseudo metric on $X$, we shall refer to the pair $(X, d)$ as a pseudo metric space. Recall:

2.1. Definition. ([8],[9]) $A$ partial metric is a function $\sigma: X \times X \rightarrow[0, \infty)$ such that for all $x, y, z \in X$ :

(i) $x=y \Leftrightarrow \sigma(x, x)=\sigma(x, y)=\sigma(y, y)$;

(ii) $\sigma(x, x) \leq \sigma(x, y)$;

(iii) $\sigma(x, y)=\sigma(y, x)$;

(iv) $\sigma(x, z) \leq \sigma(x, y)+\sigma(y, z)-\sigma(y, y)$.

For partial metric $\sigma$ on $X$, the pair $(X, \sigma)$ will be called a partial metric space. Note that for all $x$ and $y$ in a partial metric space $(X, \sigma), \sigma(x, y)=0$, imply that $x=y$. The converse does not necessarily hold, also, the value $\sigma(x, x)$ usually referred to as the size or weight of $x$, is a feature used in applications, for instance to describe the amount of information contained in $x$.

A metric space is a partial metric space but not conversely.

\subsection{Example.}

Let $X=\left\{\frac{1}{n}: n \geq 1\right\}$. Define $\sigma: X \times X \rightarrow[0, \infty)$ by

$$
\sigma(x, y)=\max \{x, y\} \text {. }
$$

Then $(X, \sigma)$ is a partial metric space. The reader can easily verify that $(X, \sigma)$ is neither a pseudo metric space, nor a metric space.

The reader should note that not every pseudo metric space is a partial metric space.

\subsection{Example.}

Let $X$ be a nonempty set, such that, the cardinality of $X,|X| \geq 2$. Define $\sigma$ : $X \times X \rightarrow[0, \infty)$ by $\sigma(x, y)=0$, for all $x, y \in X$. Then $(X, \sigma)$ is a pseudo metric space but not a partial metric space.

However, the following holds:

2.1. Proposition. Let $X$ be nonempty set. A function $\sigma: X \times X \rightarrow[0, \infty)$ is a metric if and only if $\sigma$ is both a pseudo metric and a partial metric on $X$.

Let $X$ be a nonempty set and $\mathcal{U}$ be a filter on $X \times X$. We denote by $\Delta=\{(x, x): x \in$ $X$ \}. Suppose that the following hold for $X$ and $\mathcal{U}$ on $X$ :

(a) $\Delta \subset U$, for every $U \in \mathcal{U}$;

(b) For every $U \in \mathcal{U}$ there exists $V \in \mathcal{U}$ such that $V \circ V \subseteq U$. Then the filter $\mathcal{U}$ is called a quasi-uniformity [5] on $X$, and the pair $(X, \mathcal{U})$ is referred to as a quasi-uniform space. Note that a quasi-uniformity $\mathcal{U}$ is a uniformity on $X$ if and only if for every $U \in \mathcal{U}$, the inverse of $U$, denoted by $U^{-1}$ contains a member of $\mathcal{U}$. So, every uniformity on $X$ is a quasi-uniformity, but the converse is not necessarily true. 
2.1. Theorem. Let $(X, \sigma)$ be a partial metric space. The family $\left\{U_{n}: n=1,2, \ldots\right\}$ with

$$
U_{n}=\left\{(x, y) \in X \times X: \sigma(x, y)<\sigma(x, x)+\frac{1}{n}\right\}
$$

is a base for the quasi-uniformity $\mathcal{U}_{\sigma}$ induced by $\sigma$.

Proof. Let $(X, \sigma)$ be a partial metric space and $U_{n}=\{(x, y) \in X \times X: \sigma(x, y)<$ $\left.\frac{1}{n}+\sigma(x, x)\right\}$ for each $n \in \mathbb{N}$. We show that $\left\{U_{n}, n \in \mathbb{N}\right\}$ is a base for the uniformity $\mathcal{U}_{\sigma}$. Note that $\{(x, x): x \in X\} \subset U_{n}$ for each $n \in \mathbb{N}$. Since $n+1>n$, we have $U_{n+1} \subset U_{n}$. Finally, for each $n \in \mathbb{N}$ there is $m \in \mathbb{N}$ such that $U_{m} \circ U_{m} \subset U_{n}$. In particular, for each $n \in \mathbb{N}$ choose, $m>2 n$ then $\frac{1}{m}+\frac{1}{m}<\frac{1}{n}$. Let $(x, z) \in U_{m} \circ U_{m}$. The find $y \in X$ such that $(x, y) \in U_{m}$ and $(y, z) \in U_{m}$. So, from $\sigma(x, z) \leq \sigma(x, y)+\sigma(y, z)-\sigma(y, y)$ we get

$$
\sigma(x, z) \leq \frac{1}{m}+\sigma(x, x)+\frac{1}{m}+\sigma(y, y)-\sigma(y, y)=\frac{2}{m}+\sigma(x, x) .
$$

Thus $\sigma(x, z) \leq \frac{1}{n}+\sigma(x, x)$. Therefore $(x, z) \in U_{n}$, this shows that $U_{m} \circ U_{m} \subset U_{n}$. Hence the set $\left\{U_{n}: n \in \mathbb{N}\right\}$ is a base for the quasi-uniformity $\mathcal{U}_{\sigma}$ on $X$.

2.1. Remark. Although in a partial metric space $(X, \sigma)$, we have $\sigma(x, y)=\sigma(y, x)$ for all $x, y \in X$, we note that $\sigma(x, y)<\sigma(x, x)+\epsilon$, for $\epsilon>0$, does not necessarily mean that $\sigma(x, y)<\sigma(y, y)+\epsilon$, or conversely. This means that the sets $U_{n}$ in Theorem 2.1 are not necessarily symmetric, hence the quasi uniformity generated by the partial metric $\sigma$ is not necessarily a uniformity.

We recall that a topological space $(X, \tau)$ is quasi-metrizable if there is a quasi metric $d$ on $X$ such that $\tau=\tau_{d}$. In this case we say that $d$ is compatible with $\tau$, and that $\tau$ is a quasi-metrizable topology. Furthermore, given a partial metric space $(X, \sigma)$, the set $\left\{B\left(x, \frac{1}{n}\right)\right\}$ form a base for the topology $\tau_{\sigma}$, where $B\left(x, \frac{1}{n}\right)=\{y \in X: \sigma(x, y)<$ $\left.\sigma(x, x)+\frac{1}{n}\right\}$.

2.1. Corollary. For every partial metric space $(X, \sigma)$, the topological space $\left(X, \tau_{\sigma}\right)$, is quasi-metrizable.

Proof. Let $(X, \sigma)$ be a partial metric space and $U_{n}=\{(x, y) \in X \times X: \sigma(x, y)<$ $\left.\frac{1}{n}+\sigma(x, x), n \geq 1\right\}$. Then the set $\left\{U_{n}: n \in \mathbb{N}\right.$ form a countable base for the quasi uniformity $\mathcal{U}_{\sigma}$. Now for each $x \in X$, and each $n=1,2, \ldots, U_{n}(x)=\{y \in X: \sigma(x, y)<$ $\left.\sigma(x, x)+\frac{1}{n}\right\}=B\left(x, \frac{1}{n}\right)$. So that the topology induced by the quasi uniformity $\mathcal{U}_{\sigma}$ coincides with $\tau_{\sigma}$. Hence by Theorem 2.1 the quasi uniform space $\left(X, \mathcal{U}_{\sigma}\right)$ is quasi-metrizable. This completes the proof.

\section{Main Results}

The following definition is given in [4]

3.1. Definition. Let $E$ be an ordered vector space. An element $e \in E$ is called an order unit if for each $x \in E$ there exists $\lambda \in \mathbb{R}$ such that $x<\lambda$ e.

Let $E$ be an ordered Banach space and $K$ be subset of $E$. Note that $K$ is a cone if $K$ is closed with respect to the norm-topology, and for nonnegative scalars $\alpha, \beta$, we have $\alpha x+\beta y \in K$, for $x, y \in K$, also, for a zero vector $\theta$ in $E, x \in K$, and $-x \in K$, imply that $x=\theta$. A cone $K$ is normal, if $x \leq y$ in $K$ implies that there exists a constant $M>0$ such that $\|x\| \leq M\|y\|$, with $M$ being a minimal such constant, and $\leq$ is a partial ordering on $E$ induced by the cone $K$. That is for $x, y \in E$ we write $x \leq y$, if $y-x \in K$.

3.2. Definition. [13] Let $X$ be a nonempty set and $E$ an ordered Banach space with a cone $K$ in $E$ such that $\operatorname{int}(K) \neq \emptyset$ and $K$ closed. A function $\sigma_{c}: X \times X \rightarrow E$ is a partial cone metric if for $x, y, z \in X$ :

(i) $\theta \leq \sigma_{c}(y, y)=\sigma_{c}(x, y)=\sigma_{c}(x, x)$ if and only if $x=y$; 
(ii) $\sigma_{c}(x, y)=\sigma_{c}(y, x)$ for all $x, y$;

$($ iii $) \sigma_{c}(x, z) \leq \sigma_{c}(x, y)+\sigma_{c}(y, z)-\sigma_{c}(y, y)$ for all $x, y, z$. In this case $\left(X, E, K, \sigma_{c}\right)$ is called a partial cone metric space.

\subsection{Example.}

Let $E=\mathbb{R}^{2}, K=\{(x, y) \in E: x, y \geq 0\}$, and $X=\mathbb{N}$. Define $\sigma_{c}: X \times X \rightarrow E$ by

$$
\sigma_{c}(x, y)=\left(\max \left\{\frac{1}{x}, \frac{1}{y}\right\}, \alpha \cdot \max \{x, y\}\right),
$$

with $\alpha>0$. Then $\left(X, E, K, \sigma_{c}\right)$ is not a partial metric space but a partial cone metric space. Also, $\left(X, E, K, \sigma_{c}\right)$ is not a cone metric space.

3.3. Definition. [12] Let $X$ be a nonempty set, $E$ be an ordered vector space with a cone $K$. The function $d: X \times X \rightarrow E$ is called a quasi-cone metric if for $x, y, z \in X$ :

(i) $\theta \leq d(x, y)$ for all $x, y$;

(ii) $d(x, y)=\theta=d(y, x)$ if and only if $x=y$;

(iii) $d(x, y) \leq d(x, z)+d(z, y)$ for all $x, y, z$. In this case $(X, E, K, d)$ is called a quasicone metric space.

Note that $\left(X, E, K, d^{-1}\right)$ is also a quasi-cone metric space, where $d^{-1}: X \times X \rightarrow E$ is defined by $d^{-1}(x, y)=d(y, x)$, for all $x, y \in X$. The function $d^{-1}$ is usually called a conjugate of $d$ it is also a quasi-cone metric on $X$. Now for all $x, y \in X$, let $d^{s}(x, y)=$ $d(x, y)+d^{-1}(x, y)$. Then the function $d^{s}: X \times X \rightarrow E$ is a cone metric on $X$.

\subsection{Example.}

Let $E=\mathbb{R}^{2}$ and $X=\mathbb{R}$. Put

$$
K=\{(x, y) \in E: x, y \geq 0\}
$$

For $\alpha \geq 0$, we define

$$
d: X \times X \rightarrow E
$$

by $d(x, y)=((x-y), \alpha(x-y))$ when $x \geq y$ and $d(x, y)=(0,0)=\theta$, otherwise. Then $(X, E, K, d)$ is a quasi-cone metric space.

3.1. Proposition. [13] Let $\left(X, E, K, \sigma_{c}\right)$ be a partial cone metric space. The function $d: X \times X \rightarrow E$ defined by

$$
d(x, y)=\sigma_{c}(x, y)-\sigma_{c}(x, x)
$$

for all $x, y \in X$ is a quasi-cone metric on $X$.

In the sequel, given a partial cone metric space $\left(X, E, K, \sigma_{c}\right)$ we denote the associated quasi-cone metric space by $(X, E, K, d)$.

For a partial cone metric space $\left(X, E, K, \sigma_{c}\right)$, define

$$
B_{<<}(x, e)=\left\{y \in X: \theta<<e+\sigma_{c}(x, x)-\sigma_{c}(x, y)\right\}
$$

and let $A \subset X$. We shall say $A$ is an open subset in $X$ if for every $a \in A$, there exists $u$ where $\theta<<u$ such that say $B_{<<}(a, u) \subseteq A$.

3.1. Theorem. Let $\left(X, E, K, \sigma_{c}\right)$, be a partial cone metric space. For each $x \in X$, and $e \in \operatorname{int}(K)$ the subset $B_{<<}(x, e)$ is open in $X$.

Proof. Let $b \in B_{<<}(x, e)$. Then $\theta<e+\sigma_{c}(x, x)-\sigma_{c}(x, b)$, we put $u=e+\sigma_{c}(x, x)-$ $\sigma_{c}(x, b)$. So, $\theta<<u$. Now let $c \in B_{<<}(b, u)$. Then $\theta<u+\sigma_{c}(b, b)-\sigma_{c}(b, c)$. So $\theta \ll$ $u+\sigma_{c}(b, b)-\sigma_{c}(b, c)$. Actually, $\sigma_{c}(x, c) \leq \sigma_{c}(x, b)+\sigma_{c}(b, c)-\sigma_{c}(b, b)$. Hence,

$$
\sigma_{c}(x, c) \leq \sigma_{c}(x, b)+u+\sigma_{c}(b, b)-\sigma_{c}(b, b) .
$$


From $u=e+\sigma_{c}(x, x)-\sigma_{c}(x, b)$ we get

$$
\sigma_{c}(x, c)<e+\sigma_{c}(x, x) .
$$

Thus,

$$
\theta \ll e+\sigma_{c}(x, x)-\sigma_{c}(x, c) .
$$

It follows that $c \in B_{<<}(x, e)$, so $B_{<<}(b, u) \subseteq B_{<<}(x, e)$. This shows that $B_{<<}(x, e)$ is open.

3.2. Proposition. Let $\left(X, E, K, \sigma_{c}\right)$, be a partial cone metric space and e $\in \operatorname{int}(K)$ be fixed. Then the family $\left\{B_{<<}(x, r e): x \in X, \theta<<r\right\}$ forms a basis for the topology.

Proof. Let $u \in \operatorname{int}(K)$, be an order unit. For some $r \in(0, \infty)$, we have re $\leq u$, hence $r e+\sigma_{c}(x, x) \leq u+\sigma_{c}(x, x)$. Thus $\theta \ll u+\sigma_{c}(x, x)$ implies $\theta \ll r e+\sigma_{c}(x, x)$. It follows that $B_{<<}(x, r e) \subset B_{<<}(x, u)$.

Given a partial cone metric space $\left(X, E, K, \sigma_{c}\right)$, let us denote the topology induced by $\sigma_{c}$ with $\tau_{\sigma_{c}}$, and the topology induced by the associated quasi cone metric $d$ by $\tau_{d}$. .

3.2. Theorem. Let $\left(X, E, K, \sigma_{c}\right)$, be a partial cone metric space. Then there exists a quasi uniformity $\mathcal{U}$ of $X$ such that $\tau_{\mathcal{U}}=\tau_{\sigma_{c}}$.

Actually, we have:

3.1. Lemma. Let $\left(X, E, K, \sigma_{c}\right)$, be a partial cone metric space and $e \in \operatorname{int}(K)$ be fixed. Then $\left\{U_{\sigma_{c}, \frac{1}{n} e}\right\}$ is a countable base for a quasi-uniformity $\mathcal{U}$ on $X$.

Proof. Let $x \in X$ and define $U_{\sigma_{c}, \frac{e}{n}}[x]=\left\{y \in X: \theta<<\frac{e}{n}+\sigma_{c}(x, x)-\sigma_{c}(x, y)\right\}$. We will show that the collection $\left\{U_{\sigma_{c}, \frac{e}{n}}\right\}$ is a base for a quasi uniformity on $X$. The proof is similar to that of Theorem 2.1. We first note that for each $\frac{e}{n}, n=1,2, \ldots \Delta \subset U_{\sigma_{c}, \frac{e}{n}}$. We have $U_{d, \frac{e}{n+1}} \subset U_{\sigma_{c}, \frac{e}{n}}$. Let $n$ and $m$ be positive integers such that $2 n<m$. We will show that $U_{\sigma_{c}, \frac{e}{m}} \circ U_{\sigma_{c}, \frac{e}{m}} \subset U_{\sigma_{c}, \frac{e}{n}}$. Let $(x, y) \in U_{\sigma_{c}, \frac{e}{m}} \circ U_{\sigma_{c}, \frac{e}{m}}$. Find $z \in X$ such that $(x, y) \in U_{\sigma_{c}, \frac{e}{m}}$ and $(y, z) \in U_{\sigma_{c}, \frac{e}{m}}$. Clearly, $\theta \ll \frac{2 e}{m}+\sigma_{c}(x, x)-\sigma_{c}(x, z)$ hence $\theta \ll \frac{e}{n}+\sigma_{c}(x, x)-\sigma_{c}(x, z)$. Since $(x, z) \in U_{\sigma_{c}, \frac{e}{m}} \circ U_{\sigma_{c}, \frac{e}{m}}$, this shows that $(x, z) \in$ $U_{\sigma_{c}, \frac{e}{n}}$. It follows that $\left\{U_{\sigma_{c}, \frac{e}{n}}\right\}$ is a countable base for the quasi uniformity $\mathcal{U}$ on $X$.

We conclude this section by strengthening our earlier result, namely, Proposition 3.1

3.3. Theorem. Let $\left(X, E, K, \sigma_{c}\right)$, be a partial cone metric space and $e \in \operatorname{int}(K)$ be fixed. Then the topological space $\left(X, \tau_{\sigma_{c}}\right)$ is quasi-metrizable.

Proof. Let $\left(X, E, K, \sigma_{c}\right)$, be a partial cone metric space, $e \in \operatorname{int}(K)$ be fixed and $\mathcal{U}$ be the quasi uniformity induced on $X$ as obtained by Lemma 3.1. The quasi uniformity $\mathcal{U}$ has a countable base, and the topology induced by the quasi uniformity $\mathcal{U}$ coincides with the topology induced by $\sigma_{c}$. Let $d$ be as in Proposition 3.1 then we have $\tau_{\sigma_{c}}=\tau_{d}=\tau_{\mathcal{U}}$. Hence $\left(X, \tau_{\sigma_{c}}\right)$ is quasi-metrizable.

Actually, let $\left(X, E, K, \sigma_{c}\right)$ be a partial cone metric space and $(X, E, K, d)$ be a quasi cone metric space. The function $\rho_{d}: X \times X \rightarrow[0, \infty)$ defined by

$$
\rho_{c}(x, y)=\inf \{\|u\|: d(x, y) \ll u\}
$$

is a quasi-metric on $X$, where $u \in K$, and $\theta \ll u$.

\section{Contractions and fixed point theory}

Let $\left(X, E, K, \sigma_{c}\right)$, be a partial cone metric space and $\left\{x_{n}\right\}$ be a sequence in $X$. The sequence $\left\{x_{n}\right\}$ converges to a point $x \in X$ if $\lim _{n} \sigma_{c}\left(x_{n}, x\right)=\sigma_{c}(x, x)=\lim _{n} \sigma_{c}\left(x_{n}, x_{n}\right)$.

Let $\left(X, E, K, \sigma_{c}\right)$ be a partial cone metric space. A sequence $\left\{x_{n}\right\}$ is referred to as a $\ll-u-$ Cauchy, where $\theta \ll u$, if for every $\epsilon>0$ there exists $k$ such that $\sigma_{c}\left(x_{m}, x_{n}\right) \ll \epsilon u$ 
for all $m, n \geq k$. The sequence $\left\{x_{n}\right\}$ is $\ll-$ Cauchy if it is $\ll-u-$ Cauchy for all $u \in E$ with $\theta \ll u$. We shall say that the sequence $\left\{x_{n}\right\}$ is $\ll-u-$ convergent to $x$ is for each $\epsilon>0$ there exists $k$ such that $\sigma_{c}\left(x_{n}, x\right) \ll \epsilon u$ for all $n \geq k$, where $\theta \ll u$. The sequence $\left\{x_{n}\right\}$ is $\ll-$ converging to $x$ if it $\ll-u-$ converges to $x$ for all $u \in E$, with $\theta \ll u$. We say that $\left(X, E, K, \sigma_{c}\right)$ is $\ll-$ Cauchy complete if every $\ll-u$ - Cauchy sequence converges to a point $x \in X$ and $\sigma_{c}(x, x)=\theta$.

Following the work of R. H. Haghi et al in [6], we note that for every partial cone metric space $\left(X, E, K, \sigma_{c}\right)$ there is an associated cone metric space $\left(X, E, K, d_{c}\right)$, where the cone metric $d_{c}: X \times X \rightarrow E$ is defined by $d_{c}(x, y)=\sigma_{c}(x, y)$, if $x \neq y$ and $d_{c}(x, y)=\theta$, otherwise. We note the following:

a) The sequence in a partial cone metric space $\left(X, E, K, \sigma_{c}\right)$ that $\ll-$ converges to a point $x$ also converges to $x$ but the converse is not necessarily true, see Example 4.1 .

b) In partial metric spaces the notions of $\ll-$ completeness and $0-$ completeness coincide.

c) In cone metric spaces the notions of $\ll-$ completeness and completeness coincide.

4.1. Proposition. Let $\left(X, E, K, \sigma_{c}\right)$ be a partial cone metric space. Suppose that a sequence $\left\{x_{n}\right\} \ll-$ converges to $x$ and $y$. Then $x=y$.

Proof. Suppose that $\left\{x_{n}\right\}$ converges to both $x$ and $y$. Since $\sigma_{c}(x, x) \leq \sigma_{c}(x, y)$ for all $x, y \in X$, we will show that $\sigma_{c}(x, y) \leq \sigma_{c}(x, x)$ and $\sigma_{c}(x, y) \leq \sigma_{c}(y, y)$. To do this we observe that if a sequence in a cone partial metric space $\left(X, E, K, \sigma_{c}\right) \ll-$ converges to a point then it converges to the point, also, $\sigma_{c}(x, y) \leq \sigma_{c}\left(x, x_{n}\right)+\sigma_{c}\left(x_{n}, y\right)-\sigma_{c}\left(x_{n}, x_{n}\right)$. Taking $n \rightarrow \infty$ and since $\lim _{n} \sigma_{c}\left(x_{n}, x_{n}\right)=\sigma_{c}(x, x)$, we get $\sigma_{c}(x, y) \leq \sigma_{c}(x, x)$. Now $\sigma_{c}(x, x) \leq \sigma_{c}(x, y)$ for all $x, y \in X$. So $\sigma_{c}(x, x)=\sigma_{c}(x, y)$, hence, $\sigma_{c}(x, y)=\sigma_{c}(x, x)=$ $\sigma_{c}(y, y)$. It follows that $x=y$.

Let $\left(X, E, K, \sigma_{c}\right)$ be a partial cone metric space. The following is easy and therefore we omit the proof.

4.2. Proposition. Let $\left(X, E, K, \sigma_{c}\right)$ be a partial cone metric space. Then the following hold:

a) If $\left\{x_{n}\right\}$ is a $\ll-$ Cauchy sequence in $\left(X, E, K, \sigma_{c}\right)$ then it is a Cauchy sequence in the cone metric space $\left(X, E, K, d_{c}\right)$.

b) If $\left(X, E, K, \sigma_{c}\right)$ is $\ll-$ complete then the cone metric space $\left(X, d_{c}\right)$ is complete.

Let $\left(X, E, K, \sigma_{c}\right)$, be a partial cone metric space and $\left(X, E, K, d_{c}\right)$ be the associated cone metric space. A map $T:\left(X, E, K, \sigma_{c}\right) \rightarrow\left(X, E, K, \sigma_{c}\right)$ is a contraction whenever

$$
\sigma_{c}(T x, T y) \leq \alpha \sigma_{c}(x, y)
$$

for all $x, y \in X$ and $\alpha \in[0,1)$. Note that if $T:\left(X, E, K, \sigma_{c}\right) \rightarrow\left(X, E, K, \sigma_{c}\right)$ is a contraction, then

$$
d_{c}(T x, T y) \leq \alpha d_{c} a(x, y)
$$

holds for all $x, y \in X$ and $\alpha \in[0,1)$. We also note that given a cone metric space there is an equivalent metric space [3]. So as an application, recall:

4.1. Theorem. For each complete metric $\sigma: X \times X \rightarrow[0, \infty)$, and every ( self map) function $T: X \rightarrow X$ such that for all $x, y \in X$,

$$
\sigma(T x, T y) \leq c \sigma(x, y)
$$

with $c \in[0,1)$ there exists a unique $a \in X$, such that $a=T a$. 
4.2. Theorem. Let $\left(X, E, K, \sigma_{c}\right)$, be a $\ll-$ Cauchy complete partial cone metric space and $K$ be a normal cone. Suppose that a mapping $T: X \rightarrow X$ satisfies the contractive condition

$$
\sigma_{c}(T x, T y) \leq c \sigma_{c}(x, y),
$$

for all $x, y \in X$ where $c \in[0,1)$ is a constant. Then there exists a point $a \in X$ such that $a=T a$ and for any $x \in X$ the iterative sequence $\left\{T^{n} x\right\}$ converges to $a$.

Proof. Let $\left(X, E, K, \sigma_{c}\right)$, be a - Cauchy complete partial cone metric space. Then the associated cone metric space $\left(X, E, K, d_{c}\right)$ is complete. Now let $(X, \rho)$ be the the equivalent metric space /[3], [4],[6]], so that $(X, \rho)$ is complete. The function $T:(X, \rho) \rightarrow$ $(X, \rho)$ is a contraction whenever $T:\left(X, E, K, \sigma_{c}\right) \rightarrow\left(X, E, K, \sigma_{c}\right)$ is a contraction. Hence by Theorem 4.1, for $T:(X, \rho) \rightarrow(X, \rho)$ there exists $a \in X$ such that $a=T a$ and the sequence $\left\{T^{n}\right\}$ converges to $a$.

It is worth mentioning the given a partial cone metric space $\left(X, E, K, \sigma_{c}\right)$, the space $\left(X, E, K, \sigma_{c}\right)$ may be $\ll-$ Cauchy complete, without the cone metric space $\left(X, E, K, d^{s}\right)$ being complete, with $d^{s}(x, y)=2 \sigma_{c}(x, y)-\sigma_{c}(x, x)-\sigma_{c}(y, y)$. Actually an example of a $\ll-$ Cauchy complete partial cone metric space with the cone metric space $\left(X, E, K, d^{s}\right)$ which is not complete is found in [10]. So generally in the proof of Theorem 4.2 we cannot replace the cone metric space $\left(X, E, K, d_{c}\right)$ with $\left(X, E, K, d^{s}\right)$.

\section{Acknowledgment}

We would like to thank the anonymous referees whose report helped in the improvement of this manuscript. Finally, the support from South African National Research Foundation(NRF, Grant Number 92052) and Tshwane University of Technology(TUT) through Department of Mathematics and Statistics are acknowledged.

\section{References}

[1] T. Abdeljawad, Quasi cone metric spaces and generalizations of Caristi Kirk's theorem, Fixed Point Theory and its Applications, 9 pages, ID 574387, 2009.

[2] M. Asadi, B.E. Rhoades, H. Soleimani, Some note on the paper "The equivalence of cone metric spaces and metric spaces, Fixed point theory and applications, 87, 1- 4, 2012.

[3] W-S. Du, A note on cone metric fixed point theory and its equivalence, Nonlinear Anal., 72, 2259 - 2261, 2010.

[4] Z. Ercan, On the end of the cone metric spaces, Topology and its Applications, 166, 10-14, 2014.

[5] P. Fletcher, W. F. Lindgren, Quasi uniform spaces, Marcel Dekker Inc., New York, 1982.

[6] R. H. Haghi, Sh. Rezapour, N. Shahad, Be careful on partial metric fixed point results, Topopoly and its Applications 160, 450-454, 2013.

[7] X. Ge, S. Lin, Completions of partial metric spaces, Topology and its Applications, 182, 16-23, 2015.

[8] R. Heckmann, Approximation of metric spaces by partial metric spaces, Appl. Cat. Struct. 7, $71-83,1999$.

[9] S. G. Matthews, Partial metric topology, in: Proceedings of the $8^{\text {th }}$ Summer Conference on General Topology and its Applications, Ann. New York Acad. Sci. 728, 183-196, 1994.

[10] S. Romaguera, A Kirk type characterization of completeness for partial metric spaces, Fixed Point Theory and its Applications, vol 2010, Article ID 493298,6 pages, 2010.

[11] S. Romaguera, O. Valero, A quantitative computational model for complete partial metric spaces via formal balls, Mathematical Structures in Computer Science, 19, 541-563, 2009.

[12] F. Shadda, M. S. MD Noorani, Fixed point results in quasi cone metric spaces, Abstract and Applied Analysis, Vol 2013, Article ID 3036267 pages, 2013.

[13] A. Sönmez, Fixed point theorems in partial cone metric space, arXiv:1101.2741v1, 2011. 
